\title{
Characterization of spatial light distribution of flash lamp systems
}

\author{
by F. Cernuschi', M.Lamperti ${ }^{2}$, R. Marchesi ${ }^{3}$, A. Russo ${ }^{3}$ \\ 1 ENEL S.p.A- Centro di Ricerca Ambiente e.Materiali, Via Rubattino, 5420132 Milano Italy; \\ ${ }^{2}$ Università degli Studi di Milano-Istituto di Fisica Generale Applicata, Via Celoria, 1620133 Milano \\ Italy; \\ ${ }^{3}$ Politecnico di Milano- Dipartimento di Energetica, P.za Leonardo da Vinci, 3220133 Milano Italy
}

\begin{abstract}
In this work results of an experimental activity are reported, where we evaluated the spatial distribution of light intensity for two different flash lamp systems.

Two different methods have been experimented; in the first one a map of the energy distribution was obtained using a lab energy-meter while in the second one the temperature distribution of a homogeneous flat surface few instants after being flashed was imaged using an IR camera.

A comparison between the spatial features of the two flash systems and between the two methods is done.
\end{abstract}

\section{Forward}

NDE active thermographic techniques have found wide applications in the detection of defects within materials close to the surface [1]. The main applications concern the assessment of the structural integrity of coated components.

These techniques are based on the IR detection (by using a thermographic system) of the inhomogeneities (related to the presence of a sub-surface defects) of surface temperature distribution produced by a uniform heating due to a thermal radiant source. This source may be pulsed or modulated. In dependence of the type of the radiating source the thermographic techniques are defined as follows: Video Pulsed Thermography (VPT) $[2,3]$ and Lock-in Thermography (LT) [4].

VPT performances in detecting sub-surface defects can be influenced by the characteristics of the heating source, typically a flash lamp system. In particular, both flash duration and light spatial distribution play a keyrole in the surface thermal gradient (contrast) across a defect. To optimise the detection of defects the spatial distribution of the lighting thermal source is required as uniform and intense as possible.

In order to investigate the influence of the above parameters on the induced thermal field a characterization of the spatial distribution of two different systems has been carried out.

\section{Experimental}

\subsection{Experimental set-up}

For the characterization, we used a commercial photographic flash lamp (A) and a prototype flash lamp system (B). The two systems differ in the flash tube colour temperature, in the geometry of tubes, in energy, and in the surface finish of the parabolic reflectors. In table 1 the main features of both systems are reported.

Two different measuring methods have been used; in the first one a direct measurement of the emitted radiation has been performed by detecting the incident energy in different points of a defined grid laying on a plane perpendicular to the direction of lamp emission. The features of the energy meter (a thermopile) used for the tests are the following: range $400 \mathrm{~nm}-1100 \mathrm{~nm}$; minimum energy resolution $1 \mathrm{~mJ}$; effective sensitive area $25 \mathrm{~mm}^{2}$; collimation angle $22^{\circ}$. The measurement plane was chosen at one meter 
from the lamps (see figure 1). Two mutual perpendicular rotation stages allowed to rotate the detector in order to compensate misalignments between lamp and collimator of the detector. The defined grids were chosen in dependence of the different geometry of the two characterised flash lamps.

Table 1

\begin{tabular}{|l|l|l|}
\hline parameters & A & B \\
\hline Gas within the tube & Xenon & Xenon \\
\hline Tube colour temperature [K] & 5600 & 9300 \\
\hline Tube geometry & annular toroidal & linear \\
\hline Pulse duration [ms] & 10 & 3 \\
\hline Geometry of reflector & rotational paraboloid & $\begin{array}{l}\text { cylindrical } \\
\text { paraboloid }\end{array}$ \\
\hline Reflector surface finish & rough & specular \\
\hline
\end{tabular}

The second method refers to an indirect detection of the emitted radiation by infrared thermography. In this case a black bristol board sheet was placed on the measurement plane (20 cm away from the lamps) and the temperature distribution induced on the whole plane by the incident radiation was imaged by a thermographic system placed on the opposite side of the lamps as shown in figure 2. Black bristol board offers three advantages related to: its thin thickness, its low thermal diffusivity and high efficiency in the conversion incident radiation to heat. Main features of the infrared camera are the following: range $8-12 \mu \mathrm{m}$, instantaneous field of view $1.8 \mathrm{mrad}$, noise equivalent temperature difference $0.1^{\circ} \mathrm{C}$, minimum resolvable temperature difference $0.2^{\circ} \mathrm{C}$, frame rate 25 frame/s.

\subsection{Experimental measurement procedure}

\subsubsection{Direct method}

In each position of the detector, the energy value was obtained averaging four flashes. A suitable correction was performed in order to take into account the applied rotation of the detector in respect of its normal position. As a matter of fact the needed rotations of the detector modify the energy density incident on the active area of detector. In order to obtain a bidimensional map of the energy distribution, experimental data have been interpolated by using the Kriging gridding method that is widely applied in geostatistical sciences [5]. Final results for both lamps systems are reported in figure 3.

\subsubsection{Indirect method}

Both lamps were placed at 20 centimeters away from the paper sheet while the thermocamera was at a distance of 1.9 meters from the black bristol board sheet. Infrared images of the thermal distribution (induced by the flash) on the rear side of the paper sheet were selected at times at which the temperature in the hottest region reached its maximum.

Figure 4 shows infrared images of the two systems

\section{Discussion and conclusions}

Comparing experimental data of the two lamps obtained by using the direct method it seems that the emitted energy in the central region is similar for both systems. On the contrary, thermal images of the two flash lamps show that lamp $A$ is more effective in heating the bristol board. This can be explained taking into account that the lamps differ in their optical spectrum. As a matter of fact the two lamps differ in the tube colour temperature and the higher the temperature the higher is the low wavelength (UV in this 


\section{http://dx.doi.org/10.21611/qirt.1996.003}

case) content in the emitted radiation. Since UV is not effective in heating dielectric materials like bristol board [6], lamp $B$ (which has the higher tube colour temperature) appears to be less energetic by the indirect measurement in respect of the direct one.

Furthermore the IR content of the emitted radiation, especially by the lamp A (due to its lower colour temperature) is not detected by the energy-meter (because it is out of the sensitivity range) but its effect in heating is well imaged by the IR camera. Both effects contribute to the already mentioned differences. A detailed analysis of these effects and a study aimed at a quantitative evaluation of the emitted energy by flash systems have been recently started.

\section{REFERENCES}

[1] MALDAGUE (X.). - Nondestructive Evaluation of Materials by Infrared Thermography. London, Springer-Verlag, 224 p.,1993.

[2] KUO (P.K.), AHMED (T.), FAVRO (L.D.), JIN (H.J.), THOMAS (R.L.). - Synchronous Thermal IR Video Imaging for Nondestructive Evaluation, Jour. of Nondestructive Evaluation, Vol. 8, $n^{\circ} 2,1989$, p.97-106.

[3] CROWTHER (D.J.), FAVRO (L.D.), KUO (P.K.), THOMAS (R.L.). Analytical calculation and numerical simulations of box-car thermal wave images of planar subsurface scatterers. Thompson (D.O.), Chimenti (D.E.) eds., Review of Progress in Quantitative Nondestructive Evaluation, Vol.11, 1992, Plenum Press, p. 417-423.

[4] BUSSE (G.), WU (D.), KARPEN (W.). - Thermal wave imaging with phase sensitive modulated thermography. J. Appl. Phys. 71, 8, 1992, p. 3962-3965.

[5] CRESSIE, (N.A.C.). - The origins of Kriging. Mathematical Geology, Vol.22, 1990, p. 239-252.

[6] ALMOND (D.P.), PATEL (P.M.). - Photothermal science and techniques. London, Chapman \&Hall, 241p. 1996.

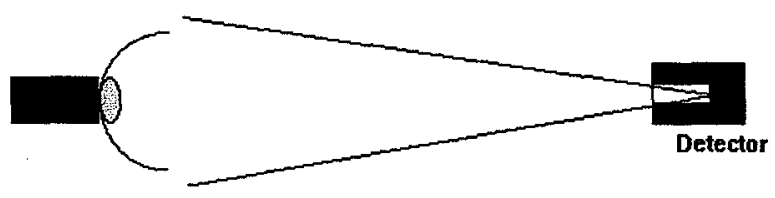

Flash Iamp

Fig.1 A Schematic view of the experimental set-up of the direct method.
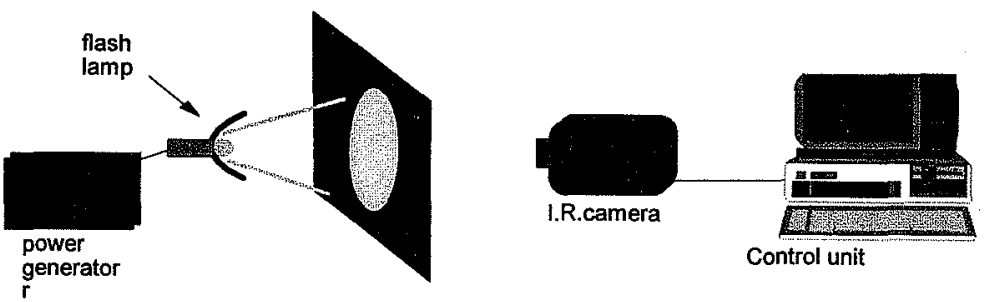

Fig.2 A Schematic view of the experimental set-up of the indirect method. 


\section{http://dx.doi.org/10.21611/qirt.1996.003}

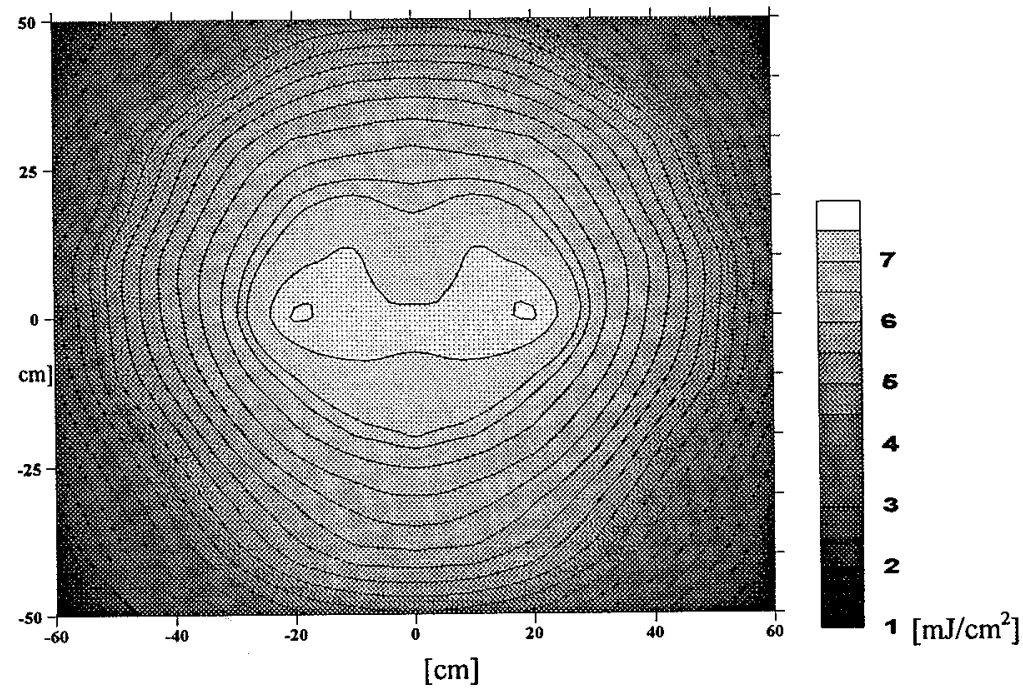

(a)

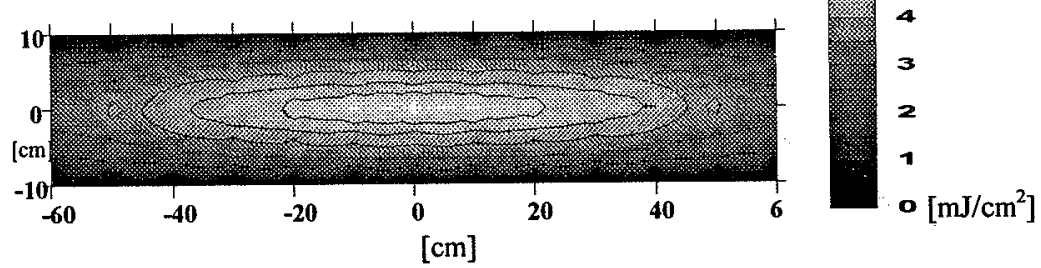

(b)

Fig. 3 Bidimensional distribution of the emitted energy from lamp A (a) and lamp B (b) respectively. 


\section{http://dx.doi.org/10.21611/qirt.1996.003}
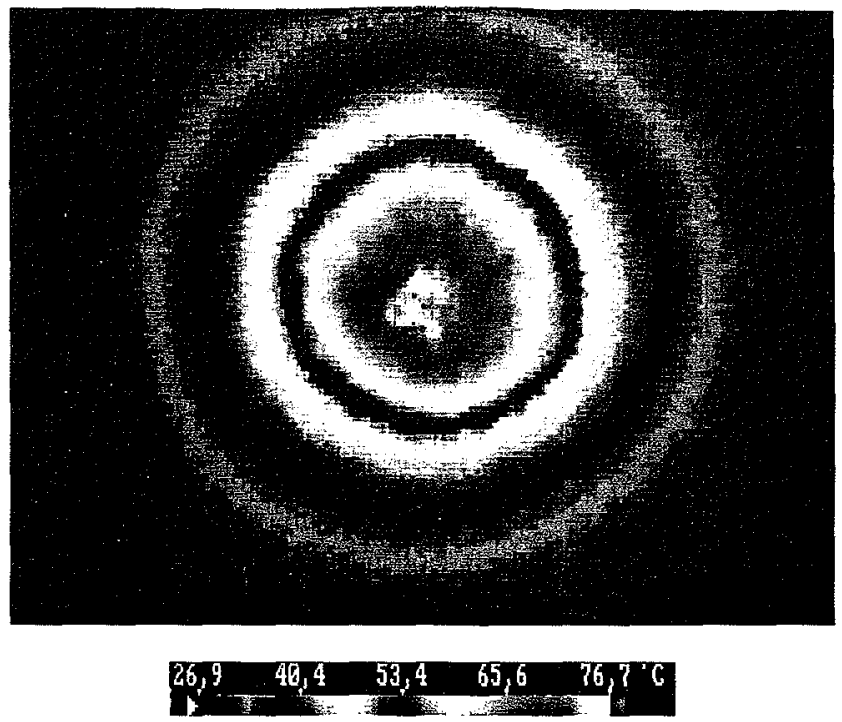

(a)
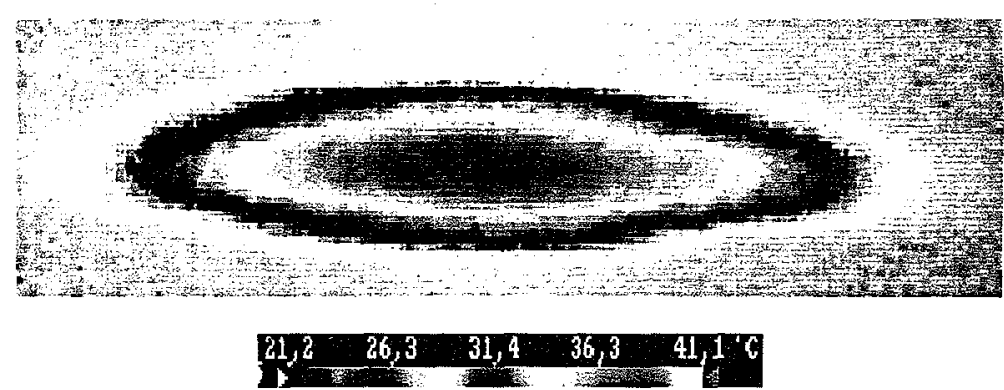

(b)

Fig.4 IR images of the temperature distribution on by the bristol board after flashes produced by lamp $A$ (a) and lamp B (b) respectively. 